\title{
TTR
}

Traduction, terminologie, re?daction

\section{Tartarin chez les Anglos : intraduisibilité et pertinence}

\section{Claude Romney}

Volume 8, numéro 2, 2e semestre 1995

Technolectes et dictionnaires

URI : https://id.erudit.org/iderudit/037224ar

DOI : https://doi.org/10.7202/037224ar

Aller au sommaire du numéro

Éditeur(s)

Association canadienne de traductologie

ISSN

0835-8443 (imprimé)

1708-2188 (numérique)

Découvrir la revue

Citer cet article

Romney, C. (1995). Tartarin chez les Anglos : intraduisibilité et pertinence. TTR, 8(2), 211-225. https://doi.org/10.7202/037224ar

\section{Résumé de l'article}

Tartarin chez les Anglos : intraduisibilité et pertinence - Cet article applique les concepts de la théorie de la pertinence de Sperber et Wilson à la comparaison de cinq traductions anglaises et américaines de Tartarin de Tarascon d'Alphonse Daudet. Pour que deux messages ou textes se ressemblent, il faut, selon cette théorie, qu'ils comportent des explicatures et implicatures semblables. De plus, la compréhension ne doit exiger qu'un effort minime d'assimilation. Nous avons utilisé ces concepts pour analyser les différentes solutions apportées par les traducteurs comme équivalents des méridionalismes et des éléments provençaux que contient le texte de Daudet. Certaines traductions américaines conservent tels quels un nombre relativement élevé de termes étrangers et la théorie de la pertinence appliquée à la traduction permet de voir que ce maintien comporte des avantages.

Tous droits réservés ( $\odot$ TTR: traduction, terminologie, rédaction — Les auteurs, Ce document est protégé par la loi sur le droit d'auteur. L’utilisation des 1995 d'utilisation que vous pouvez consulter en ligne.

https://apropos.erudit.org/fr/usagers/politique-dutilisation/ 


\section{Tartarin chez les Anglos: intraduisibilité et pertinence}

\section{Claude Romney}

On s'accorde à présent pour reconnaître que les traductions sont caractéristiques de l'époque de leur production et que, si elles varient, c'est «not because they are good or bad, but because they have been produced to satisfy different demands» (Bassnett et Lefevere, 1990, p. 5). De fait, ces mêmes auteurs sont d'avis que «translations are made to respond to the demands of a culture, and of various groups within that culture» (p. 7).

Il n'est donc pas surprenant qu'un certain nombre de versions américaines ou anglaises de Tartarin de Tarascon (Daudet, 1987 [1872]) dont la publication s'échelonne entre 1887 et 1968 présentent des traits divers selon la date et le lieu de leur parution. L'une d'entre elles (Daudet, 1900), due à une Américaine, Katharine Prescott Wormeley, offre une particularité qui saute littéralement aux yeux puisqu'elle contient, se détachant en italique, un nombre élevé de mots et d'expressions conservés en français ou en provençal. On peut se demander en quoi ce maintien, qui résulte certainement d'une décision consciente de la part de la traductrice, est commandé par les goûts et les besoins des lecteurs américains de la fin du $\mathrm{XIX}^{\mathrm{e}}$ siècle. Pourquoi les autres traductions, anglaises comme américaines, que nous avons eu l'occasion d'examiner, ne renferment-elles pas autant de mots et d'expressions gardés tels quels? C'est à cette question que nous allons tenter de répondre, en nous appuyant sur la théorie de la pertinence appliquée à la traduction. 
Il convient d'abord de dire deux mots de la place de Daudet dans l'histoire littéraire et également de caractériser Tartarin de Tarascon. Alphonse Daudet n'est plus considéré de nos jours que comme un auteur secondaire de la fin du $\mathrm{XIX}^{\mathrm{e}}$ siècle, ce qui peut paraitre étonnant, étant donné la haute estime en laquelle ses contemporains le tenaient, aussi bien en France qu'à l'étranger. Parmi ses admirateurs les plus célèbres, on peut citer Flaubert, Zola et Henry James, ce dernier l'ayant qualifié d' «admirable genius». Pour sa part, Robert Louis Stevenson considérait Daudet comme de loin le meilleur des romanciers français de l'époque (Matthews, 1901). Un journal londonien l'appelait «the French comic giant» et, dès leur publication, ses romans firent l'objet de très nombreuses traductions, notamment en anglais et américain, en allemand et en russe.

On place d'ordinaire Daudet parmi les écrivains régionalistes. Né à Nîmes en 1840, ami de Mistral et des félibres, poètes et écrivains de langue d'oc, il a en effet pris pour cadre de plusieurs de ses romans et nouvelles la Provence qu'il a décrite parfois de façon attendrissante, mais dont il s'est aussi gentiment moqué, en particulier dans Tartarin, ce dont les Tarasconnais, atteints dans leur fierté, lui ont longtemps gardé rancune.

Daudet, comme le souligne Louis Michel dans l'étude qu'il a consacrée au langage méridional dans son œuvre (1961), écrivait pour le public parisien et c'était à dessein qu'il employait des méridionalismes. Selon Michel (p. 19): «Linguistiquement parlant, un méridionalisme est un tour ou un mot propre au Midi de la France» et «au point de vue littéraire [...], c'est un terme qui dépayse le lecteur parisien et l'entraîne en imagination vers le Midi.» De temps en temps, Daudet ne se privait pas non plus d'utiliser des termes à proprement parler provençaux pour rehausser encore davantage la couleur locale. Les traducteurs ont donc dû prendre une décision à propos de ces méridionalismes et ces vocables provençaux, caractéristiques de Tartarin: les garder tels quels en français ou en provençal, les traduire littéralement ou les transposer en anglais à l'aide d'expressions qui leur paraissaient fonctionnellement équivalentes. Ils devaient aussi se poser une question semblable au sujet d'un certain nombre de termes originaires d'Afrique du Nord, donc en fait arabes, qu'on trouve dans la seconde moitié du livre. En effet, l'action se transporte alors avec 
Tartarin en Algérie, qu'il appelait «chez les Teurs», c'est-à-dire les Turcs, ses notions de géographie étant plutôt vagues, et où il avait l'ambition d'aller à la chasse au lion. Nous nous limiterons dans la présente étude au cas des méridionalismes et des tournures provençales, mais il est bien entendu que le même genre de remarques pourrait se faire à propos des autres expressions perçues comme étrangères en français standard.

Une des rares spécialistes qui se soient penchés sur les problèmes de la traduction des dialectes est Godeleine Carpentier, dans un article fort intéressant intitulé "Traduire la forme, traduire la fonction: la représentation du dialecte dans deux genres littéraires» (1990). En opposant la fonction à la forme, cette auteure reprend d'ailleurs en partie des idées déjà exprimées par Mildred L. Larson dans Meaning-based Translation: A Guide to Cross-Language Equivalence (1984, pp. 163-166) et, avant elle, par Juliane House (1981), selon laquelle il est désirable que la fonction du texte cible corresponde à celle du texte source. C'est à cette dernière que nous empruntons la définition de la fonction: «[T]he function of a text is the application [...] or use which the text has in the particular context of a situation" (p. 37). Godeleine Carpentier est d'avis de proscrire les emprunts purs et simples conservés dans la langue source parce qu'ils ne sont pas intelligibles aux lecteurs de la traduction (nous allons voir que cette affirmation est d'ailleurs discutable). De plus, selon elle, «la traduction littérale et ponctuelle de la forme conduit trop souvent à un manque d'authenticité [...] quand ce n'est pas à un charabias pur et simple» (p. 82). Le choix d'un dialecte de la langue cible n'est pas indiqué non plus car les connotations sont incompatibles avec le contenu culturel du texte source. D'après Godeleine Carpentier, il convient donc de privilégier la fonction par rapport à la forme, en tenant compte principalement de la fonction d'information du texte à traduire. Dans le cas qui nous occupe, et dans la perspective de la thèse de Carpentier qui n'est certes pas la seule façon d'envisager les problèmes de traduction des dialectes, il serait par conséquent souhaitable de prendre en considération la portée des éléments méridionaux et provençaux, c'est-à-dire le double effet qu'ils produisaient et produisent encore sur le lecteur parisien ou du moins sur le lecteur français non-méridional. Quand nous disons double effet, c'est qu'il s'agissait d'une part d'un élément de couleur locale, puisque le lecteur était entraîné en imagination vers le Midi, comme le 
disait Michel (1961), et également d'un effet comique, puisque bon nombre de ces expressions étaient employées par l'auteur pour tourner en dérision le Méridional, en principe typique, qu'était Tartarin.

La notion de l'équivalence des "fonctions» en traduction, relativisée par les théories récentes de la littérature et de la traduction, peut sans doute prêter le flanc aux critiques. Elle cadre cependant bien avec les concepts de la pragmatique et, de plus, répétons-le, rares sont les études qui se soient penchees sur la question des dialectes en traduction ${ }^{1}$, bien que certains auteurs, comme Hatim et Mason (1990, pp. 40-41), aient reconnu son importance. C'est pourquoi les remarques de Carpentier nous ont semblé dignes d'intérêt.

Exposons à présent brièvement certains principes de la théorie de la pertinence, en anglais relevance theory, dus aux linguistes Sperber et Wilson et appliqués à la traduction par Ernst-August Gutt dans son livre Translation and Relevance: Cognition and Context (1991). La pertinence d'un texte dépend essentiellement de deux facteurs. D'une part, ce sont les effets contextuels (à noter le sens particulier donné à contexte qui renvoie à la connaissance du monde ou aux «présupposés» - en anglais assumptions - faites par les communicateurs à son sujet, c'est-à-dire à leur «environnement cognitif»); il ne s'agit donc nullement d'éléments extérieurs aux colocuteurs, mais de concepts cognitifs. En outre, ce qui est désirable dans un acte communicatif, c'est qu'il n'exige qu'un minimum d'effort d'assimilation de la part du récepteur du message. Comme le souligne Gutt,

[t]he central claim of relevance theory is that human communication crucially creates an expectation of optimal relevance, that is an expectation on the part of the hearer that his attempt at interpretation will yield adequate contextual effects at minimal processing cost ( $\mathrm{p}$. 30; les italiques sont de l'auteur).

D'autre part, dans un acte de communication, l'interprétation dépendra des explicatures et des implicatures. Les explicatures, ce sont «those

1. Paru à la fin de 1994, le volume VII, $\mathrm{n}^{\circ} 2$, de TTR («Traduire les sociolectes») dirigé par Annick Chapdelaine et Gillian Lane-Mercier est l'un de ces rares ouvrages d'ensemble sur cette problématique. 
analytical implications which the communicator intends to convey», tandis que les implicatures sont «the contextual assumptions which he intends to convey» (Gutt, 1991, p. 38). Pour que deux messages ou textes se ressemblent, comme par exemple un original et sa traduction, il faut qu'ils partagent les mêmes explicatures et implicatures. Ces concepts de la théorie de la pertinence vont nous servir pour l'analyse des solutions apportées par les traducteurs à certains problèmes de traduction de Tartarin.

Les traductions de Tartarin de Tarascon que nous avons eu l'occasion d'examiner sont les suivantes':

1) la plus ancienne, une traduction anonyme publiée à Londres par Routledge en 1887 , soit quinze ans après la parution de la première édition française, a été reprise en 1954 par Dent à Londres et Dutton à New York, avec la mention erronée «1st edition 1910»;

2) une traduction américaine, également anonyme, publiée à New York par l'éditeur Crowell en 1895;

3) une autre traduction américaine, due à Katharine Prescott Wormeley et publiée à Boston par Little, Brown et Cie en 1900;

4) une traduction anglaise sans date, due à A.K.P. Wingate et publiée à Londres par Collins, probablement vers 1920;

5) une traduction anglaise de J.M. Cohen, beaucoup plus récente, puisque publiée en 1968 par la Folio Society à Londres. Cohen, traducteur chevronné, était aussi l'auteur d'une histoire de la traduction.

Les deux traducteurs américains, et surtout Wormeley, ont laissé subsister en français ou en provençal un certain nombre de mots ou expressions de l'original. Quels sont-ils? Nous avons essayé de les classer, mais, pour chaque catégorie, nous ne pourrons évidemment présenter que quelques exemples typiques que nous examinerons à la lumière de la théorie de la pertinence.

On trouve d'abord dans la traduction de Wormeley, ainsi qu'à un moindre degré dans celle de l'édition Crowell, un grand nombre d'exclamations conservées telles qu'on les trouve dans le texte source. Ces exclamations ou jurons soit appartiennent au français standard soit

2. Ces versions seront désignées du nom du traducteur ou, pour celles qui sont anonymes, du nom du premier éditeur. 
sont perçus comme des régionalismes. Parmi les exclamations ou jurons du français standard, on relève mon Dieu, utilisé par Wormeley (p. 6) dans «Hey! mon Dieu! [the hunters from Tarascon] go out into the open country.» Parbleu (p. 66) et pardi (p. 102) sont également conservés par la traductrice américaine.

$\mathrm{Au}$ nombre des régionalismes, on trouve le juron populaire méridional coquin de sort, très commun selon Michel (p. 119). Euphémisme pour «coquin de Dieu», «il est de toutes les colères, de toutes les surprises, de tous les mouvements d'humeur, qu'il calme, qu'il apaise». Daudet l'utilise tout au début du livre pour souligner le contraste de l'intérieur de la maison de Tartarin et son aspect extérieur. Le juron est conservé tel quel par Crowell (p. 3): «But when you stepped inside, coquin de sort, [...]» et également par Wormeley (p. 1).

Parmi les autres jurons typiques du Midi de la France, on trouve dans la traduction de Crowell pécaïré à la page 15 (variante de péchère). Boun diou (p. 54) est un juron à proprement parler provençal que Wormeley a aussi maintenu. De même qu'elle a gardé tel quel l'euphémisme tron de l'er dans «Be quiet, tron de l'er!» (p. 54), en se méprenant d'ailleurs plus loin (p. 125) sur le sens de tron dans Tron de Diou, qu'elle rend par "Throne (of God)», alors qu'il s'agit de «tonnerre de Dieu».

La remarque principale qu'on peut faire à propos du maintien dans un texte anglais ou américain de ces jurons français et provençaux, c'est qu'ils ne se distinguent plus alors les uns des autres: les implicatures, évidentes pour le lecteur français, disparaissent puisque ces termes ne font pas partie de l'environnement cognitif de la plupart des lecteurs de la traduction, incapables de distinguer entre français et provençal. L'effet produit est un renforcement de l'élément d'étrangeté, au sens de foreignness, c'est-à-dire presque d'exotisme, mais d'un exotisme qui confond le fait français et le fait méridional. Le caractère régional de l'œuvre s'en trouve atténué et, en même temps, son ton comique, étant donné que, pour les Français du Nord, l'accent du Midi comporte toujours des connotations légèrement amusantes.

D'un autre côté, la présence du point d'exclamation à la suite du juron indique clairement la valeur de l'expression qui apparaît donc 
comme accessoire et non essentielle à la compréhension, même si le lecteur de la traduction n'en saisit pas le sens exact. L'erreur de sens commise par Wormeley sur tron de Diou semble alors sans importance.

D'autres traductions, comme celle de Routledge, ont plutôt naturalisé les expressions exclamatives et les jurons. Par exemple, coquin de sort est rendu par l'expression cocasse "ye gods and little fishes» (p. 1), et pécaïré par une autre exclamation amusante, «odsbodikins» (p. 10) (corruption de "God's body» suivi du suffixe diminutif «-kin»), ce qui fait que par le sens ce juron correspondrait davantageà parbleu, bien que les connotations soient différentes puisque ce juron français n'a rien de comique. Les implicatures des équivalents choisis par le traducteur anonyme de Routledge sont donc semblables à celles des jurons méridionaux de Daudet: les exclamations feront sourire les lecteurs de langue anglaise qui ne leur attacheront cependant aucune origine géographique particulière. Le principe de la pertinence est respecté, puisque les effets contextuels sont obtenus moyennant un coût modique de traitement, mais en partie seulement puisque les implicatures renvoyant à un lieu géographique disparaissent.

Par contre, quand J.M. Cohen traduit coquin de sort par «Lord bless my soul» (p. 13), boun diou simplement par «in God's name» (p. 54) et tron de l'er par «for God's sake» (p. 55), les implicatures ne sont plus du tout les mêmes que celles suggérées par l'original, puisque, contrairement à ce qui se passe en français, les expressions anglaises choisies ne sont pas perçues comme des régionalismes, pas plus qu'elles ne sont comiques, surtout les deux dernières. Du point de vue de la pertinence et en ce qui concerne cet aspect particulier du texte, la traduction de Cohen, bien que plus moderne, est donc moins adroite.

En second lieu, on peut examiner des termes ayant trait à la nourriture. Ainsi saucissot (selon Michel (p. 62) méridionalisme qui correspond à (saucisson») est conservé par Wormeley (p. 6), dans une énumération des victuailles qu'emportaient les chasseurs de Tarascon lors de leurs expéditions dominicales: "un beau morceau de bœuf en daube, des oignons crus, un saucissot (en italique dans le texte français), quelques anchois» (p. 79). Etant donné la ressemblance de saucissot avec l'anglais "sausage», le lecteur de langue anglaise comprendra aisément le sens. De plus, celui qui connaît le mot «saucisson» du 
français standard se rendra peut-être compte qu'il s'agit d'une variété régionale. Donc, d'une certaine manière, la décision de Wormeley de conserver le mot étranger dans sa traduction n'est pas aussi bizarre qu'elle le paraitrait au premier abord. Les implicatures et même les explicatures sont plus proches de celles du texte français que dans les versions anglaises qui traduisent par «sausage», comme celle de Routledge (p. 5), renvoyant au monde contextuel des Anglais, pour lesquels a sausage a un aspect et un goût totalement différents de ceux d'un «saucisson" pour un Français. La traduction de Cohen qui propose comme équivalent «black pudding» (p. 17) s'éloigne encore davantage du français non seulement sur le plan des explicatures mais aussi des implicatures. En effet, il est peu probable que le «black pudding», plat qui n'est pas spécialement régional, entre dans la composition d'un menu de pique-nique, contrairement au saucisson ou saucissot.

Autre expression intéressante, eau bouillie, que Wormeley ( $\mathrm{p}$. 32) conserve telle quelle, en la faisant suivre de la traduction de l'explication fournie par Daudet, ce qui donne: "What is called eau bouillie in Tarascon consists of slices of bread steeped in hot water with a clove of garlic, a sprig of thyme, and a pinch of bay-leaf.) Pour sa part, Cohen (p. 34) se contente de donner la description: «a broth of hot water with a clove of garlic, [etc.]", jugeant superflue l'appellation de ce genre de bouillon typiquement provençal. Mais alors l'intention de l'auteur d'attirer l'attention sur ce potage particulier à Tarascon disparaît et les effets contextuels, au sens de la théorie de la pertinence, ne sont plus suffisants.

Passons assez rapidement sur des termes divers maintenus en français et dont beaucoup désignent des personnes. Ainsi, «a few lorettes» (Wormeley, p. 58), sans explication, et «two cocottes rejoining their regiments» (p. 95). Les lectrices américaines comprenaient-elles vraiment qui étaient ces lorettes et ces cocottes? Ou l'intention de la traductrice, s'érigeant alors en censeur, était-elle de faire en sorte que le sens leur échappe? La signification de «squadrons of chasseurs d'Afrique» (p. 60) est plus explicite.

Un terme qui mérite cependant qu'on s'y attache davantage est celui de lapin, employé par le "brave commandant Bravida» pour qualifier Tartarin: «C'est un lapin!». Et l'auteur d'ajouter: «et vous 
pensez si le commandant s'y connaissait en lapins, après en avoir tant habillé» (p. 84). Le jeu de mots n'est plus guère à la portée des francophones de la fin du XX $\mathrm{XX}^{\mathrm{e}}$ siècle, mais selon l'édition du Littré de 1874 (contemporain de Tartarin), le qualificatif de lapin «se [disait] familièrement d'un homme brave, vigoureux.» De plus, l'expression «il est brave comme un lapin' s'employait à propos d'une personne habillée de neuf. On comprend alors que le commandant Bravida, chargé de l'habillement des nouvelles recrues, s'y soit connu en lapins de cette sorte. Les explicatures se perdent complètement sous la plume de Wormeley qui se contente de reproduire le terme français, comme elle le fait souvent: "and you may well think the commander was knowing in lapins, having clothed so many of them» (p. 12), les lapins n'ayant plus, si l'on peut dire, ni queue ni tête. Il est curieux de constater que la solution adoptée par Wormeley produisait à son époque un effet analogue à celui créé par le texte original de Daudet sur les lecteurs français modernes qui ne comprennent plus le sens donné à lapin par leurs arrière-grands-parents.

La même traductrice américaine n'hésite pas non plus à proposer comme titre d'un des chapitres l'expression surprenante The Rendez-vous of the Lapins (p. 67). La traductrice accroît certes l'impression d'exotisme, mais au point de rendre l'appellation de cette buvette quasi incompréhensible. L'effort exigé pour l'interprétation du texte par le lecteur n'est plus minime, comme l'exige la théorie de la pertinence. La solution adoptée supprime de plus le jeu de mots voulu par l'auteur sur le nom de ce café algérien, lieu de rencontre des chasseurs intrépides qui, si la fortune cynégétique leur avait souri, rapportaient aussi dans leur gibecière de nombreux vrais lapins.

Les autres traducteurs proposent des équivalents divers pour lapin, allant de «buck rabbit» (Crowell, p. 23) à «sportsman» (Cohen, p. 40). Wingate emploie d'abord «fine fellow» (p. 14) pour qualifier Tartarin. Par contre, il désigne le café du nom de «the rendezvous of sportsmen» (p. 53), amenuisant ainsi le lien entre le héros et la buvette (puisque Tartarin est lui-même un lapin, il est normal qu'il se trouve au rendez-vous des lapins), et, par là même, la cohésion du texte. Dans tous ces cas, même si les explicatures correspondent plus ou moins à celles de l'original, surtout dans le cas de «sportsmen», les implicatures 
sont oblitérées, «fine fellow» et «sportsmen» n'impliquant aucun jeu de mot et aucune idée comique.

Dernière catégorie dont nous donnerons des exemples: les expressions qui reproduisent "l'accent du Midi» et que Daudet a utilisées à la fois pour accroître la couleur locale et produire un effet comique. Ainsi, dans une scène de comédie pure où Tartarin chante un duo avec $\mathrm{M}^{\text {me }}$ Bézuquet la mère, lorsque c'est son tour de chanter, notre héros «le bras tendu, le poing fermé, la narine frémissante, disait par trois fois d'une voix formidable, qui roulait comme un coup de tonnerre dans les entrailles du piano: "Non!... non!... non!...», ce qu'en bon Méridional, il prononçait: «Nan!... nan!...nan!...» Comme l'indique Michel (p. 88), «Daudet a remarqué que parfois dans la voyelle nasale on, l'o est tellement ouvert et long qu'il paraît être un $a$ s. Trois des traductions (Wormeley, p. 8; Crowell, p. 17, et Wingate, p. 13) conservent l'orthographe française de «Nan! nan! nan!» et la prononciation anglaise ou américaine détruit jusqu'aux explicatures puisque les exclamations utilisées n'ont pas de sens en anglais ou, au pire, renvoient au diminutif du prénom Nancy. Cohen (p. 18) adapte en indiquant que la prononciation normale de «No! No! No!» devient «Naw! Naw! Naw!» Laquelle de ces deux solutions est-elle la plus pertinente? Sans aucun doute, celle de Cohen qui a le mérite de conserver les explicatures et de suggérer des implicatures équivalentes: son «Naw! Naw! Naw!» sera perçu par les lecteurs de langue anglaise comme comique.

Daudet émaille aussi les dialogues de Tartarin des locutions et autrement et au moins, en expliquant que ces expressions prononcées «autremain» et «au mouain» s'utilisent au début et à la fin de chaque phrase (p. 97). Crowell (p. 58) se contente de reproduire la prononciation accompagnée de l'explication en traduction: «For at Tarascon, all phrases begin with et autrement pronounced autremain, and conclude with au moins, which is pronounced au mouain.» Wormeley adopte une solution différente et incorpore l'équivalent de sens en anglais dans la phrase suivante:

At Tarascon all sentences begin with et autrement (there pronounced autremain) and end with au moins (pronounced au mouain). On this occasion above all others, the "at leasts» and the "otherwises» resounded throughout the town till the windows rattled. (p. 30) 
Cette façon de rendre ce passage parait assez habile, mais seulement jusqu'à un certain point. Si elle permet, en effet, de se rendre compte que l'accent tarasconnais se démarque de la prononciation standard, pour le lecteur qui lit autremain et au mouain à l'anglaise ou à l'américaine, les voyelles nasales sont dénaturées. Pour conserver plus ou moins les effets contextuels, la traductrice aurait pu expliquer que, dans la bouche des habitants de Tarascon, la dernière syllabe subissait une transformation caractéristique de l'accent du Midi.

Quant à lui, Wingate (p. 26) a choisi de transposer à l'aide d'expressions anglaises équivalentes sur le plan fonctionnel: «For at Tarascon all sentences begin by and besides, which is pronounced besoides, and end with at least, pronounced laste.» Enfin, Cohen adopte le même genre de solution, mais ses équivalents fonctionnels sont Tell me et $I$ suppose, prononcés respectivement «termi» et «Is'pose» (p. 34). Ainsi, ces deux traducteurs traduisent les expressions (ce qui a évidemment pour effet de les acclimater), comptant sans doute sur la prononciation populaire ainsi que sur la répétition pour produire un effet comique. L'accent méridional se transforme dans la plus ancienne de ces deux traductions en accent aux résonances irlandaises. La plus récente propose une prononciation plutôt populaire, sans qu'elle soit définie géographiquement. C'est pourquoi la première de ces solutions répond davantage aux critères de la pertinence: l'effort d'interprétation exigé est très faible et les explicatures comme les implicatures sont adéquates, l'accent irlandais ayant pour les Anglais des connotations comiques.

Que conclure de tout cela? La première observation est que les traductions britanniques se distinguent des traductions américaines examinées en ce qu'elles ont tendance à "naturaliser» le texte de Daudet. Certes, l'effort d'interprétation que doit fournir le lecteur est alors très faible, mais on obtient souvent une inadéquation des explicatures comme des implicatures. Le Tartarin de ces versions, et davantage peut-être celui recréé par Cohen, perd une bonne partie de son aspect comique de Méridional et son langage manque souvent de couleur, de pittoresque.

Les traductions américaines, presque centenaires à présent, se permettent, nous l'avons vu, de conserver un certain nombre de 
tournures en français et en provençal. La théorie de la pertinence appliquée à la traduction permet cependant de voir que ce maintien de termes étrangers comporte des avantages. Lorsqu'il s'agit de jurons suivis d'un point d'exclamation, de termes dont l'aspect permet de les rattacher à un mot anglais les rendant à peu près compréhensibles, ainsi que d'éléments d'une énumération, dont le sens approximatif peut se déduire de celui des autres, l'effort d'interprétation n'est pas énorme.

Ajoutons à ces remarques une hypothèse concernant le lectorat des traductions américaines du tournant du siècle. La France en général et particulièrement son Midi jouissaient alors de la faveur des Américains fortunés de la classe de ceux que met en scène dans ses romans Henry James, précisément grand admirateur de Daudet. Auriant, dans une étude consacrée à Quatre héros d'Alphonse Daudet (1948, p. 41), mentionne ces riches touristes d'Outre-Atlantique qui, de passage à Tarascon, alors important nœud ferroviaire, demandaient souvent à visiter la maison de Tartarin. On est en droit de supposer que c'est grâce aux traductions publiées dans leur pays qu'ils connaissaient l'existence du héros comique de Daudet. Habitués qu'ils étaient aux voyages, la présence de termes étrangers dans une traduction ne les gênait sans doute pas et ne devait leur demander que cet effort minime d'assimilation dont il est question dans la théorie de la pertinence. Pour ceux qui n'étaient jamais allés en France, Katherine Prescott Wormeley avait en général pris davantage de soin que ses confrères à bien choisir les mots ou expressions conservés en français de façon à ce que leur maintien ne nuise pas véritablement à la compréhension du roman de Daudet.

Si les traducteurs anglais éprouvaient, semble-t-il, plus de scrupules à conserver des termes étrangers dans leur texte, il est curieux de constater que dans $A$ Year in Provence, ouvrage récent (1991) [1989] qui a remporté un succès remarquable des deux côtés de l'Atlantique, l'écrivain américain Peter Mayle utilise exactement le même procédé que Wormeley pour renforcer la couleur locale. Les lecteurs originaires aussi bien du Royaume-Uni que d'Amérique du Nord comprennent sans difficulté la signification d'expressions ressemblant à l'anglais telles que résidence secondaire (p. 7), prix déclaré et affaire intéressante (p. 66), ou scandaleux (p. 70). Parfois, Mayle choisit d'utiliser des mots français dont il est facile à un anglophone de déduire le sens à partir du 
contexte: «It is the politicians, the millionaires, the grosses légumes in Paris» (p. 70) ou "I'm fine [...] but I think the car is foutu» (p. 77). La technique qu'il emploie est donc très semblable à celle de Wormeley: dans la plupart des cas, les explicatures sont nettes, bien qu'il s'agisse de mots étrangers, et les implicatures produisent l'effet de pittoresque souhaité, sans dépayser outre mesure. Tout comme Daudet, Mayle attire l'attention sur la prononciation distinctive du français par les Provençaux («demain became demang, vin became vang, maison became mesong») (p. 7). Du point de vue du vocabulaire, cependant, son livre ne comprend que très peu de mots provençaux. A Year in Provence serait donc dans une certaine mesure l'équivalent moderne de Tartarin en traduction, transportant en pensée son lecteur dans une Provence qu'il connaît déjà ou qu'il aura envie de connaître, mais ne lui permettant pas véritablement d'établir, à partir de la langue qu'on y parle, de distinction entre cette région et le reste de la France.

Claude Romney, Department of French, Italian and Spanish, The University of Calgary, 2500 University Drive N. W., Calgary, Alberta, Canada T2N 1 N4.

Courriélec: Jcromney@acs.ucalgary.ca

\section{Références}

AURIANT (1948). Quatre héros d'Alphonse Daudet. Paris, Mercure de France.

BASSNETT, Susan et André Lefevere, dir. (1990). Translation History and Culture. Londres, Pinter.

CARPENTIER, Godeleine (1990). "Traduire la forme, traduire la fonction: la représentation du dialecte dans deux genres littéraires», in Michel Ballard, dir., La Traduction plurielle. Lille, Presses Universitaires de Lille, pp. 71-92.

DAUDET, Alphonse (1987)[1872]. Tartarin de Tarascon. Paris, Presses Pocket. 
(1887). The Prodigious Adventures of Tartarin of Tarascon. [Traduction anonyme.] Londres, Routledge.

(1895). Tartarin of Tarascon. [Traduction anonyme.] New York et Boston, Crowell.

(1900). Tartarin of Tarascon to which is added Tartarin on the Alps. [Traduction de Katharine Prescott Wormeley.] Boston, Little, Brown \& Co.

(s. d.). Tartarin of Tarascon and Le Petit Chose. [Traduction de A.K.P. Wingate.] Londres et Glasgow, Collins. (1968). Tartarin of Tarascon. [Traduction de J.M. Cohen.] Londres, Folio Society.

GUTT, Ernst-August (1991). Translation and Relevance - Cognition and Context. Oxford, Blackwell.

HOUSE, Juliane (1981). A Model for Translation Quality Assessment. Tübingen, Narr.

LARSON, Mildred L. (1984). Meaning-based Translation: A Guide to Cross-Language Equivalence. Lanham (Maryland), University Press of America.

HATIM, Basil et Ian Mason (1990). Discourse and the Translator. Londres, Longman.

MATTHEWS, Brander (1901). The Historical Novel and Other Essays. New York, Charles Scribner's Sons.

MAYLE, Peter (1991) [1989]. A Year in Provence. New York, Knopf. MICHEL, Louis (1961). Le Langage méridional dans l'auvre d'Alphonse Daudet. Paris, D'Artrey. 
RÉSUMÉ: Tartarin chez les Anglos: intraduisibilité et pertinenceCet article applique les concepts de la théorie de la pertinence de Sperber et Wilson à la comparaison de cinq traductions anglaises et américaines de Tartarin de Tarascon d'Alphonse Daudet. Pour que deux messages ou textes se ressemblent, il faut, selon cette théorie, qu'ils comportent des explicatures et implicatures semblables. De plus, la compréhension ne doit exiger qu'un effort minime d'assimilation. Nous avons utilisé ces concepts pour analyser les différentes solutions apportées par les traducteurs comme équivalents des méridionalismes et des éléments provençaux que contient le texte de Daudet. Certaines traductions américaines conservent tels quels un nombre relativement élevé de termes étrangers et la théorie de la pertinence appliquée à la traduction permet de voir que ce maintien comporte des avantages.

ABSTRACT: Tartarin in Angloland: Untranslatability and Relevance - This article makes use of the concepts of Sperber and Wilson's relevance theory to compare five English and American translations of Tartarin de Tarascon by Alphonse Daudet. In order for two messages or two texts in different languages to correspond, they must, according to relevance theory, share similar explicatures and implicatures. Moreover, the effort required to process a speech act must be minimal. We have applied those concepts to the analysis of the various solutions chosen by the translators as equivalents for words and expressions characteristic of Southern France and Provence, found in Daudet's text. Some of the American translations maintain a relatively high number of foreign terms. Relevance theory applied to translation shows that there are advantages to keeping those expressions in the original language. 